\title{
Feller Property for a Special Hybrid Jump-Diffusion Model
}

\author{
Jinying Tong \\ School of Science, Donghua University, Shanghai 201620, China \\ Correspondence should be addressed to Jinying Tong; jytong@dhu.edu.cn \\ Received 13 December 2013; Accepted 15 February 2014; Published 23 April 2014 \\ Academic Editor: Litan Yan
}

Copyright (C) 2014 Jinying Tong. This is an open access article distributed under the Creative Commons Attribution License, which permits unrestricted use, distribution, and reproduction in any medium, provided the original work is properly cited.

We consider the stochastic stability for a hybrid jump-diffusion model, where the switching here is a phase semi-Markovian process. We first transform the process into a corresponding jump-diffusion with Markovian switching by the supplementary variable technique. Then we prove the Feller and strong Feller properties of the model under some assumptions.

\section{Introduction}

Stability of stochastic differential equations with Markovian switching has received a lot of attention. Assume that $(X(t), r(t))$ is a two-component Markov process such that $X(\cdot)$ is a continuous component taking values in $R^{n}$ and $r(\cdot)$ is a Markov process taking values in a finite set. More specially, the process $(X(t), r(t))$ can be described by

$$
d X(t)=f(X(t), t, r(t)) d t+g(X(t), t, r(t)) d B(t) .
$$

Mao [1] investigated the exponential stability for general nonlinear stochastic differential equations with Markovian switching. Shaikhet [2] took the time delay into account and considered the stability of a semilinear stochastic differential delay equation with Markovian switching, while Mao et al. [3] investigated the stability of a nonlinear stochastic differential delay equation with Markovian switching.

Most of these papers are concerned with asymptotic stability in probability or in mean square (i.e., the solution will tend to zero in probability or in mean square). However, this asymptotic stability is sometimes too strong and in this case it is useful to know whether or not the solution will converge in distribution (not necessary to converge to zero). Hence Wee [4] studied the stability in distribution for jumpdiffusions (without Markovian switching), whereas Yuan and Mao [5] made many corresponding researches for continuous Itô diffusions with Markovian switching. Zhang and Chen [6] considered a general condition for existence and uniqueness of stationary distribution for diffusion systems.
In addition, $\mathrm{Xi}[7]$ recently discussed the Feller continuity of a general nonlinear stochastic differential equation with Markovian switching. Later, Xi [8] further considered the Feller continuity and exponential ergodicity for a jumpdiffusion equation with Markovian switching of the form

$$
\begin{aligned}
d X(t)= & b(X(t), r(t)) d t+\sigma(X(t), r(t)) d B(t) \\
& +\int_{R^{d} \backslash\{0\}} c(X(t-), r(t-), u) N(d t, d u),
\end{aligned}
$$

where $\sigma(x, k)$ is $(d \times d)$-matrix valued and $b(x, k)$ and $c(x, k, u)$ are $R^{d}$ valued for $x, u \in R^{d}$ and $k \in N . B(\cdot)$ is a $d$ dimensional standard Brownian motion and $N(\cdot)$ is a Poisson measure.

Note that these papers referred to above are all concerned with Markovian switching systems. However, we know that Markovian switching systems have many limitations in applications. For example, the jump time of a Markov chain is, in general, exponentially distributed. And it is well known that the pervasiveness of the exponential distribution in stochastic systems is rarely due to empirical evidence in support of their assumption, but far more so to the ease of conditioning which results from the lack of memory property. Hence the results obtained on these systems are conservative. Besides, it is worthy to recall the phase type distribution which is a generalization of exponential distribution while still preserving much of its analytic tractability. The phase type distribution is very important in real application since the matrix-analysis method developed by Neuts [9] 
Motivated by the analysis above, our aim in this paper is to establish some criteria on the stochastic stability for a kind of more general jump-diffusions with phase semi-Markovian switching. The rest of the paper is organized as follows. In Section 2, we begin with the review of certain notions for phase type distribution and phase semi-Markov process. And then, through the supplementary variable technique, we make a transformation for the process considered in this paper. In Section 3, by using the coupling method, a proof of Feller continuity is given. Furthermore, we also prove the strong Feller continuity in Section 4.

\section{Preliminaries and Transformation}

Throughout the paper, let $(\Omega, \mathscr{F}, P)$ be a probability space supporting all the random variables and processes and $\mathscr{F}_{t}$ an increasing family of sub- $\sigma$-algebras of $\mathscr{F}$. To proceed, we will recall some definitions and properties.

Definition 1. A probability distribution $F(\cdot)$ on $[0, \infty)$ is said to be a continuous phase type distribution, if it is the distribution of the lifetime of a terminating Markov process $\{r(t)\}_{t \geq 0}$ with finitely many states and time homogeneous transition rates.

More precisely, a terminating Markov process $\{r(t)\}$ with state-space $E$ and intensity matrix $T$ is defined as the restriction to $E$ of a Markov process $\{\widehat{r}(t)\}_{0 \leq t<\infty}$ on $E_{\delta}=$ $E \cup\{\delta\}$, where $\delta$ is absorbing and the states in $E$ are transient (we often write $m$ for the number of elements of $E$ ). For any initial distribution $\alpha=\left(a_{1}, \ldots, a_{m}\right), F$ is the distribution of the time $\zeta=\inf \{t>0: \widehat{r}(t)=\delta\}$ to absorption, $F(t)=P_{\alpha}(\zeta \leq t)$. This implies in particular that the intensity matrix for $\{\widehat{r}(t)\}$ can be written in block-partitioned form as

$$
Q=\left(\begin{array}{cc}
T & T^{0} \\
\mathbf{0} & 0
\end{array}\right),
$$

where the $m \times m$ matrix $T=\left(T_{i j}\right)$ satisfies $T_{i i}<0, T_{i j} \geq 0$, $i \neq j ; T^{0}=\left(T_{1}^{0}, T_{2}^{0}, \ldots, T_{m}^{0}\right)^{T}$ is a nonnegative column vector.

The pair $(\alpha, T)$ is called the $m$ order representation of $F(\cdot)$. A basic analytical property of phase-type distribution is given by the following result.

Lemma 2. Let $F(\cdot)$ be a phase type with representation $(\alpha, T)$; then, for $t \geq 0$, the cumulative distribution function is given by

$$
F(t)=1-\alpha \exp (T t) e .
$$

Definition 3. Let $E$ be a finite or countable set. A stochastic process $\widehat{r}(t)$ on the state-space $E$ is called a phase semiMarkov process (when $E$ is finite, $\widehat{r}(t)$ is also called a finite phase semi-Markov process), if the followings hold.

(1) The sample paths of $(\widehat{r}(t) ; t<+\infty)$ are rightcontinuous step functions and have left-handed limits with probability one.
(2) Denote the $n$th jump point of the process $\widehat{r}(t)$ by $\tau_{n}(n=0,1,2, \ldots)$, where $\tau_{0} \equiv 0<\tau_{1}<\tau_{2}<\cdots<$ $\tau_{n}<\cdots, \tau_{n} \uparrow+\infty$, and the process $\widehat{r}(t)$ possesses Markov property at each $\tau_{n}(n=0,1,2, \ldots)$.

(3) $F_{i j}(t):=P\left(\tau_{n+1}-\tau_{n} \leq t \mid \widehat{r}\left(\tau_{n}\right)=i, \widehat{r}\left(\tau_{n+1}\right)=j\right)=$ $F_{i}(t)(i, j \in E, t \geq 0)$ does not depend on $j$ and $n$.

(4) $F_{i}(t)(i \in E)$ is a phase type distribution.

In the present paper, the process $(X(t), \widehat{r}(t))$ considered can be described by

$$
\begin{aligned}
d X(t)= & \widehat{b}(X(t), \widehat{r}(t)) d t+\widehat{\sigma}(X(t), \widehat{r}(t)) d B(t) \\
& +\int_{R^{d} \backslash\{0\}} \widehat{c}(X(t-), \widehat{r}(t-), u) N(d t, d u),
\end{aligned}
$$

where $\widehat{\sigma}(x, k)$ is $(d \times d)$-matrix valued and $\widehat{b}(x, k)$ and $\widehat{c}(x, k, u)$ are $R^{d}$ valued for $x, u \in R^{d}$ and $k \in E(E:=$ $\{1,2, \ldots, m\})$. Let $\widehat{r}(t)$ be a finite phase semi-Markov process on the state-space $E$, and let $B(t)$ be an $\mathscr{F}_{t}$-adapted $R^{d}$-valued Brownian motion and also a martingale with respect to $\mathscr{F}_{t}$; Let $N(d t, d u)$ be a stationary $\mathscr{F}_{t}$-Poisson point process, and let $\widetilde{N}(d t, d u)=N(d t, d u)-\Pi(d u) d t$ be the compensated Poisson random measure on $[0, \infty) \times R^{d}$, where $\Pi(\cdot)$ is a deterministic finite characteristic measure on the measurable space $\left(R^{d} \backslash\{0\}, \mathscr{B}\left(R^{d} \backslash\{0\}\right)\right)$. Assume that the Brownian motion $B(\cdot)$, the Poisson process $N(\cdot)$, and the semi-Markov process $\widehat{r}(\cdot)$ are independent from each other.

From the description above, it is easy to see that the process $(X(t), \widehat{r}(t))$ is not a Markov process, unless the sojourn time of $\widehat{r}(t)$ in each state is exponentially distributed. But we can show that considering the process $\widehat{r}(t)$ only at the jump points $\tau_{n}$ yields a (discrete-time) Markov process. Furthermore, the behaviour of $\widehat{r}(t)$ is piecewise deterministic in the intervals between jump points. For the Markovization of $(X(t), \widehat{r}(t))$ we therefore have to add the information on the neighboring jump points. Hence, to continue with our study, let $J(t)$ denote the phase of $\widehat{r}(t)$ at time $t$; then $(\widehat{r}(t), J(t))$ is a Markov process, moreover, so is process $(X(t),(\widehat{r}(t), J(t)))$.

Next, we will show some properties of Markov process $(\widehat{r}(t), J(t))$. Denote the $n$th jump point of the finite phase semi-Markov process $\widehat{r}(t)$ by $\tau_{n}(n=0,1,2, \ldots)$, where $\tau_{0} \equiv$ $0<\tau_{1}<\tau_{2}<\cdots<\tau_{n}<\cdots$. From Definition 3, we know that the staying time at each state $i \in E$ is of phase type. Hence, for each $i \in E$, correspondingly, we let $\left(\alpha^{(i)}, T^{(i)}\right)$ denote the $m^{(i)}$ order representation of $F_{i}(t)$ and $E^{(i)}$ the transient states set, where $m^{(i)}$ denotes the number of the elements in $E^{(i)}$, and

$$
\begin{aligned}
F_{i}(t) & =P\left(\tau_{n+1}-\tau_{n} \leq t \mid \hat{r}\left(\tau_{n}\right)=i\right), \quad(i \in E), \\
\alpha^{(i)} & =\left(a_{1}^{(i)}, a_{2}^{(i)}, \ldots, a_{m^{(i)}}^{(i)}\right), \\
T^{(i)} & =\left(T_{j k}^{(i)}, j, k \in E^{(i)}\right) .
\end{aligned}
$$


Let

$$
P=\left(p_{i j}, i, j \in E\right),
$$

where $p_{i j}=P\left(\widehat{r}\left(\tau_{n+1}\right)=j \mid \widehat{r}\left(\tau_{n}\right)=i\right), \quad(i, j \in E)$,

$$
(\alpha, T)=\left\{\left(\alpha^{(i)}, T^{(i)}\right), i \in E\right\} .
$$

For any $i \in E$, define

$$
\begin{aligned}
T_{j}^{(i, 0)} & =-\sum_{k=1}^{m^{(i)}} T_{j k}^{(i)} \quad\left(j=1,2, \ldots, m^{(i)}\right), \\
G & =\left\{\left(i, k^{(i)}\right) \mid i \in E, k^{(i)}=1,2, \ldots, m^{(i)}\right\} .
\end{aligned}
$$

We will see that the probability distribution of $\widehat{r}(t)$ can be determined only by $\{P,(\alpha, T)\}$.

Remark 4. The superscript of parameters $\alpha^{(i)}, T^{(i)}, m^{(i)}, E^{(i)}$, and so forth and the subscript of $F_{i}(\cdot)$ all mean that these parameters are corresponding to the state $i$.

Theorem 5. Process $r(t):=(\widehat{r}(t), J(t))$ is a Markov process with state-space $G$ ( $G$ is finite if and only if $E$ is finite). Then the infinitesimal generator of $r(t)$ given by $Q=\left(q_{\mu \nu}, \mu, v \in G\right)$ is determined only by the pair of $(\widehat{r}(t), J(t))$ given by $\{P,(\alpha, T)\}$ as follows:

$$
\begin{aligned}
& q_{\left(i, k^{(i)}\right)\left(i, k^{(i)}\right)}=T_{k^{(i)} k^{(i)}}^{(i)}, \quad\left(i, k^{(i)}\right) \in G ; \\
& q_{\left(i, k^{(i)}\right)\left(i, \bar{k}^{(i)}\right)}=T_{k^{(i)} \bar{k}^{(i)}}^{(i)} \\
& k^{(i)} \neq \bar{k}^{(i)}, \quad\left(i, k^{(i)}\right) \in G, \quad\left(i, \bar{k}^{(i)}\right) \in G \text {; } \\
& q_{\left(i, k^{(i)}\right)\left(j, k^{(j)}\right)}=p_{i j} T_{k^{(i)}}^{(i, 0)} a_{k^{(j)}}^{(j)}, \\
& i \neq j, \quad\left(i, k^{(i)}\right) \in G, \quad\left(j, k^{(j)}\right) \in G .
\end{aligned}
$$

Proof. For any $\Delta>0$, one has the following:

(1) for any $\left(i, k^{(i)}\right) \in G$, we have

$$
\begin{aligned}
& P\left\{(\widehat{r}(t+\Delta), J(t+\Delta))=\left(i, k^{(i)}\right) \mid(\widehat{r}(t), J(t))=\left(i, k^{(i)}\right)\right\} \\
& \quad=1+T_{k^{(i)} k^{(i)}}^{(i)} \Delta+o(\Delta)
\end{aligned}
$$

(2) for any $\left(i, k^{(i)}\right) \in G,\left(i, \bar{k}^{(i)}\right) \in G$, and $k^{(i)} \neq \bar{k}^{(i)}$, we have

$$
\begin{aligned}
& P\left\{(\widehat{r}(t+\Delta), J(t+\Delta))=\left(i, \bar{k}^{(i)}\right) \mid(\widehat{r}(t), J(t))=\left(i, k^{(i)}\right)\right\} \\
& \quad=T_{\left.k^{(i)}\right)^{(i)}}^{(i)} \Delta+o(\Delta)
\end{aligned}
$$

(3) for any $\left(i, k^{(i)}\right) \in G,\left(j, k^{(j)}\right) \in G$, and $i \neq j$, we have

$$
\begin{aligned}
& P\left\{(\widehat{r}(t+\Delta), J(t+\Delta))=\left(j, k^{(j)}\right) \mid(\widehat{r}(t), J(t))=\left(i, k^{(i)}\right)\right\} \\
& \quad=p_{i j} T_{k^{(i)}}^{(i, 0)} a_{k^{(j)}}^{(j)} \Delta+o(\Delta) .
\end{aligned}
$$

The proof is complete.

For the existence and uniqueness of process $X(t)$ satisfying (5), we make the following assumption.

Assumption 6. Assume that $\widehat{c}(x, i, u)$ is $\mathscr{B}\left(R^{d} \times E\right) \times \mathscr{B}\left(R^{d} \backslash\right.$ $\{0\})$ measurable function and that $\widehat{\sigma}(x, i)$ and $\widehat{b}(x, i)$ are continuously differentiable in $x$, and they satisfy the Lipschitz condition and the linear growth condition as follows. For some constant $H>0$,

$$
\begin{aligned}
& (\mathrm{A} 1)^{\prime}|\widehat{\sigma}(x, i)-\widehat{\sigma}(y, i)|^{2}+|\widehat{b}(x, i)-\widehat{b}(y, i)|^{2} \leq H|x-y|^{2}, \\
& (\mathrm{~A} 2)^{\prime}|\widehat{\sigma}(x, i)|^{2}+|\widehat{b}(x, i)|^{2} \leq H\left(1+|x|^{2}\right)
\end{aligned}
$$

for all $x, y \in R^{d}$ and $i \in E$.

Theorem 7. Under Assumption 6, system (5) has a unique solution $X(t)$.

Proof. From Theorem 9.1 in Chapter IV of Ikeda and Watanabe [10], it follows that, for each $k \in E$, there exists a unique strong solution $X_{k}(t)$ to the following stochastic differentialintegral equation:

$$
\begin{aligned}
d X_{k}(t)= & b\left(X_{k}(t), k\right) d t+\sigma\left(X_{k}(t), k\right) d B(t) \\
& +\int_{R^{d} \backslash\{0\}} c\left(X_{k}(t-), k, u\right) N(d t, d u)
\end{aligned}
$$

such that $X_{k}(0)=\xi$ is a $\mathscr{F}_{0}$-measurable random variable and $\left\{X_{k}(t): k \in E\right\}$ is a family of $\mathscr{F}_{t}$-adapted jump-diffusions. Since $N(t, A)$ and $\widehat{r}(t)$ are mutually independent, they have no common jumps almost surely. Therefore, each $\tau_{k}$ will not coincide with any jump instant of $X_{l}(t)$ for all $l \in E$ and we can construct the process $X(t)$ as follows. For any given initial condition $X(0)=\xi$, on the interval $\left[0, \tau_{1}\right]$, set

$$
X(t)= \begin{cases}X_{k}(t), & 0 \leq t<\tau_{1} \\ X_{k}\left(\tau_{1}\right), & t=\tau_{1} .\end{cases}
$$

Next, as was done in Section 9 in Chapter IV of Ikeda and Watanabe [10], set $\widetilde{\xi}=X\left(\tau_{1}\right), \widetilde{B}(t)=B\left(t+\tau_{1}\right)-B\left(\tau_{1}\right)$, and $\widetilde{p}(t)=p\left(t+\tau_{1}\right)$, where $p(t)$ is the random point function corresponding to $N(d t, d u)$. Similarly, we can determine the process $\widetilde{X}(t)$ on the time interval $\left[0, \tau_{2}-\tau_{1}\right]$ with respect to $\widetilde{\xi}$ as above. Then, define

$$
X(t)=\widetilde{X}\left(t-\tau_{1}\right), \quad t \in\left[\tau_{1}, \tau_{2}\right] .
$$

Continuing this procedure successively, $X(t)$ is determined uniquely on the interval $\left[0, \tau_{n}\right]$ for every $n$ and thus $X(t)$ is determined globally due to $\lim _{n \rightarrow \infty} \tau_{n}=+\infty$. 
Process $(X(t), r(t))$ is the associated Markov process of $(X(t), \widehat{r}(t))$. And for any $\alpha \in R^{n}, \beta \in R, i \in E$, and $(i, k) \in G$, we define functions $\sigma, b$, and $c$ as follows:

$$
\begin{gathered}
\sigma\left(\alpha,\left(i, k^{(i)}\right)\right) \triangleq \widehat{\sigma}(\alpha, i), \\
b\left(\alpha,\left(i, k^{(i)}\right)\right) \triangleq \widehat{b}(\alpha, i), \\
c\left(\alpha, \beta,\left(i, k^{(i)}\right)\right) \triangleq \widehat{c}(\alpha, \beta, i) .
\end{gathered}
$$

It is easy to show that for any $(\omega, t)$

$$
\begin{gathered}
\sigma(X(t), r(\omega, t)) \equiv \widehat{\sigma}(X(t), \widehat{r}(\omega, t)), \\
b(X(t), r(\omega, t)) \equiv \widehat{b}(X(t), \widehat{r}(\omega, t)), \\
c(X(t-), r(\omega, t-), u) \equiv \widehat{c}(X(t-), \widehat{r}(\omega, t-), u) .
\end{gathered}
$$

Consequently, we have the following result.

Theorem 8. The process defined in (5) is equivalent to the following process:

$$
\begin{aligned}
d X(t)= & b(X(t), r(t)) d t+\sigma(X(t), r(t)) d B(t) \\
& +\int_{R^{d} \backslash\{0\}} c(X(t-), r(t-), u) N(d t, d u),
\end{aligned}
$$

where functions $\sigma, b$, and $c$ are defined in (17)-(19). $r(t)$ is the associated Markov process of phase semi-Markov process $\widehat{r}(t)$ with infinitesimal generator $Q$ defined in (9), and processes $\widehat{r}(t)$, $B(t)$, and $N(t)$ are defined in process (5).

Proof. Substitute (17)-(19) into (20); then the theorem can be proved easily following the identity relation between functions $\sigma, b$, and $c$ and $\widehat{\sigma}, \widehat{b}$, and $\widehat{c}$, respectively.

Based on Assumption 6 and (17)-(19), we can easily get the following proposition.

Proposition 9. If Assumption 6 holds for functions $\widehat{\sigma}, \widehat{b}$, and $\widehat{c}$, then we have that $c(x, i, u)$ is $\mathscr{B}\left(R^{d} \times G\right) \times \mathscr{B}\left(R^{d} \backslash\{o\}\right)$ measurable function and that $\sigma\left(x,\left(i, k^{(i)}\right)\right)$ and $b\left(x,\left(i, k^{(i)}\right)\right)$ are continuously differentiable in $x$, and they satisfy the Lipschitz condition and the linear growth condition as follows: For some constant $H>0$,

(A1) $\left|\sigma\left(x,\left(i, k^{(i)}\right)\right)-\sigma\left(y,\left(i, k^{(i)}\right)\right)\right|^{2}+\mid b\left(x,\left(i, k^{(i)}\right)\right)-b(y$, $\left.\left(i, k^{(i)}\right)\right)\left.\right|^{2} \leq H|x-y|^{2}$,

(A2) $\left|\sigma\left(x,\left(i, k^{(i)}\right)\right)\right|^{2}+\left|b\left(x,\left(i, k^{(i)}\right)\right)\right|^{2} \leq H\left(1+|x|^{2}\right)$

for all $x, y \in R^{d}$ and $\left(i, k^{(i)}\right) \in G$.

In the following two sections, we turn to discuss the stochastic stability of the Markov process $(X(t), r(t))$ with state-space $R^{d} \times G$ satisfying (9) and (20).

\section{Feller Property}

Let $\langle\cdot, \cdot\rangle$ and $\nabla$ denote the inner product and the gradient operator in $R^{d}$, respectively. If $A$ is a vector or matrix, we use $A^{*}$ to denote its transpose. For $x \in R^{d}$, set $a(x, k)=$ $\sigma(x, k) \sigma^{*}(x, k)$. Define a metric $\lambda(\cdot, \cdot)$ on $R^{d} \times G \times R^{d} \times G$ as follows:

$$
\begin{aligned}
& \lambda\left(\left(x,\left(i, k^{(i)}\right)\right),\left(y,\left(j, k^{(j)}\right)\right)\right) \\
& \quad=|x-y|+d\left(\left(i, k^{(i)}\right),\left(j, k^{(j)}\right)\right),
\end{aligned}
$$

where $i \in E, k^{(i)} \in\left\{1,2, \ldots, m^{(i)}\right\}$ and

$$
d\left(\left(i, k^{(i)}\right),\left(j, k^{(j)}\right)\right)= \begin{cases}0, & \text { if } i=j \text { and } k^{(i)}=k^{(j)} \\ 1, & \text { if } i \neq j \text { or } k^{(i)} \neq k^{(j)}\end{cases}
$$

Therefore, $\left(R^{d} \times G, \lambda(\cdot, \cdot), \mathscr{B}\left(R^{d} \times G\right)\right)$ is a complete separable metric space and we then have a natural càdlàg space $D\left([0, \infty), R^{d} \times G\right)$.

Process $(X(t), r(t))$ has a generator $\mathscr{A}$ as follows. For each $\left(i, k^{(i)}\right) \in G$ and for any twice continuously differentiable functions $f(x, \cdot)$,

$$
\begin{aligned}
\mathscr{A} f\left(x,\left(i, k^{(i)}\right)\right)= & L\left(\left(i, k^{(i)}\right)\right) f\left(x,\left(i, k^{(i)}\right)\right) \\
& +\Omega\left(\left(i, k^{(i)}\right)\right) f\left(x,\left(i, k^{(i)}\right)\right) \\
& +Q f\left(x,\left(i, k^{(i)}\right)\right) .
\end{aligned}
$$

Here operators $L\left(\left(i, k^{(i)}\right)\right), \Omega\left(\left(i, k^{(i)}\right)\right)$, and $Q$ are further defined as follows:

$$
\begin{aligned}
& L\left(\left(i, k^{(i)}\right)\right) f\left(x,\left(i, k^{(i)}\right)\right) \\
& =\frac{1}{2} \sum_{l, h=1}^{d} a_{l, h}\left(x,\left(i, k^{(i)}\right)\right) \frac{\partial^{2}}{\partial x_{l} x_{h}} f\left(x,\left(i, k^{(i)}\right)\right) \\
& \quad+\left\langle b\left(x,\left(i, k^{(i)}\right)\right), \nabla_{x} f\left(x,\left(i, k^{(i)}\right)\right)\right\rangle, \\
& \Omega\left(\left(i, k^{(i)}\right)\right) f\left(x,\left(i, k^{(i)}\right)\right) \\
& =\int_{R^{d} \backslash\{o\}}\left(f\left(x+c\left(x,\left(i, k^{(i)}\right), u\right),\left(i, k^{(i)}\right)\right)\right. \\
& Q f\left(x,\left(i, k^{(i)}\right)\right) \\
& =\sum_{\left(j, k^{(j)}\right) \in G} q_{\left(i, k^{(i)}\right)\left(j, k^{(j)}\right)} f\left(x,\left(j, k^{(j)}\right)\right) .
\end{aligned}
$$

Lemma 10. Suppose that Assumption 6 holds. Then the jumpdiffusion process $(X(t), r(t))$ is nonexplosive. (For the proof please see Xi [8].) 
In the following, we prove the Feller continuity of process $(X(t), r(t))$ by using the coupling method. First, we construct a coupling $\widetilde{\mathscr{A}}$ for the generator $\mathscr{A}$ as follows:

$$
\begin{aligned}
& \widetilde{\mathscr{A}} f\left(x,\left(i, k^{(i)}\right), y,\left(j, k^{(j)}\right)\right) \\
& =\left(\widetilde{L}\left(\left(i, k^{(i)}\right),\left(j, k^{(j)}\right)\right)\right. \\
& \left.\quad+\widetilde{\Omega}\left(\left(i, k^{(i)}\right),\left(j, k^{(j)}\right)\right)+\widetilde{Q}\right) f\left(x,\left(i, k^{(i)}\right), y,\left(j, k^{(j)}\right)\right),
\end{aligned}
$$

for functions $f\left(x,\left(i, k^{(i)}\right), y,\left(j, k^{(j)}\right)\right)$ on $R^{d} \times G \times R^{d} \times G$, which are twice continuously differentiable in both $x$ and $y$ and have compact support. The couplings $\widetilde{L}, \widetilde{\Omega}$, and $\widetilde{Q}$ are, respectively, the corresponding parts of $L, \Omega$, and $Q$ in operator $\mathscr{A}$, which can be constructed as follows:

$$
\begin{aligned}
\widetilde{Q} g( & \left.\left(i, k^{(i)}\right),\left(j, k^{(j)}\right)\right) \\
= & \sum_{\left(\bar{i}, k^{(i)}\right) \in G}\left(q_{\left(i, k^{(i)}\right)\left(\bar{i}, k^{(i)}\right)}-q_{\left(j, k^{(j)}\right)\left(\bar{i}, k^{(\bar{i})}\right)}\right)^{+} \\
& \times\left(g\left(\left(\bar{i}, k^{(\bar{i})}\right),\left(j, k^{(j)}\right)\right)-g\left(\left(i, k^{(i)}\right),\left(j, k^{(j)}\right)\right)\right) \\
& +\sum_{\left(\bar{i}, k^{(\bar{i})}\right) \in G}\left(q_{\left(j, k^{(j)}\right)\left(\bar{i}, k^{(\bar{i})}\right)}-q_{\left(i, k^{(i)}\right)\left(\bar{i}, k^{(\bar{i})}\right)}\right)^{+} \\
& \times\left(g\left(\left(i, k^{(i)}\right),\left(\bar{i}, k^{(\bar{i})}\right)\right)-g\left(\left(i, k^{(i)}\right),\left(j, k^{(j)}\right)\right)\right) \\
& +\sum_{\left(\bar{i}, k^{(\bar{i})}\right) \in G}\left(q_{\left(i, k^{(i)}\right)\left(\bar{i}, k^{(i)}\right)} \wedge q_{\left(j, k^{(j)}\right)\left(\bar{i}, k^{(i)}\right)}\right) \\
& \times\left(g\left(\left(\bar{i}, k^{(\bar{i})}\right),\left(\bar{i}, k^{(\bar{i})}\right)\right)-g\left(\left(i, k^{(i)}\right),\left(j, k^{(j)}\right)\right)\right),
\end{aligned}
$$

where $g$ is a bounded function on $G \times G$. Let $\left(r(t), r^{\prime}(t)\right)$ be the Markov chain generated by the coupling operator $\widetilde{Q}$. Set $T=\inf \left\{t \geq 0: r(t)=r^{\prime}(t)\right\}$; then $r^{\prime}(t)$ will move together from $T$ onward. Next, for $\left(i, k^{(i)}\right),\left(j, k^{(j)}\right) \in G$ and $x, y \in R^{d}$, set

$$
\begin{aligned}
& a\left(x,\left(i, k^{(i)}\right), y,\left(j, k^{(j)}\right)\right) \\
& =\left(\begin{array}{cc}
a\left(x,\left(i, k^{(i)}\right)\right) & \sigma\left(x,\left(i, k^{(i)}\right)\right) \sigma^{*}\left(y,\left(j, k^{(j)}\right)\right) \\
\sigma\left(y,\left(j, k^{(j)}\right)\right) \sigma^{*}\left(x,\left(i, k^{(i)}\right)\right) & a\left(y,\left(j, k^{(j)}\right)\right)
\end{array}\right) \\
& b\left(x,\left(i, k^{(i)}\right), y,\left(j, k^{(j)}\right)\right)=\left(\begin{array}{c}
b\left(x,\left(i, k^{(i)}\right)\right) \\
b\left(y,\left(j, k^{(j)}\right)\right)
\end{array}\right) .
\end{aligned}
$$

Obviously, $a\left(x,\left(i, k^{(i)}\right), y,\left(j, k^{(j)}\right)\right)$ is nonnegative definite for all $\left(i, k^{(i)}\right),\left(j, k^{(j)}\right) \in G$ and $x, y \in R^{d}$. According to Chen and $\mathrm{Li}[11]$, the diffusion operator $\widetilde{L}\left(\left(i, k^{(i)}\right),\left(j, k^{(j)}\right)\right)$ is determined by $a\left(x,\left(i, k^{(i)}\right), y,\left(j, k^{(j)}\right)\right)$ and $b\left(x,\left(i, k^{(i)}\right)\right.$, $\left.y,\left(j, k^{(j)}\right)\right)$, which is a coupling of $L\left(\left(i, k^{(i)}\right)\right)$ and $L\left(\left(j, k^{(j)}\right)\right)$.
Using the change of variable theorem, we can rewrite the operator $\Omega(\cdot)$ as follows:

$$
\begin{aligned}
& \Omega\left(\left(i, k^{(i)}\right)\right) f\left(x,\left(i, k^{(i)}\right)\right) \\
& =\int_{R^{d} \backslash\{0\}}\left(f\left(x+z,\left(i, k^{(i)}\right)\right)-f\left(x,\left(i, k^{(i)}\right)\right)\right) \mu_{x,\left(i, k^{(i)}\right)}(d z),
\end{aligned}
$$

where

$$
\mu_{x,\left(i, k^{(i)}\right)}(A):=\Pi\left\{u: c\left(x,\left(i, k^{(i)}\right), u\right) \in A\right\}
$$

for $x \in R^{d},\left(i, k^{(i)}\right) \in G$; and $A \in \mathscr{B}\left(R^{d} \backslash\{0\}\right)$. Here $\mu_{x,\left(i, k^{(i)}\right)}(\cdot)$ actually is the jump measure of the first component of $(X(t), r(t))$. On the set of twice continuously differentiable functions $h(x, y)$ of $R^{d} \times R^{d}$ to $R$ with compact support, set

$$
\begin{aligned}
\widetilde{\Omega}\left(\left(i, k^{(i)}\right),\left(j, k^{(j)}\right)\right) h(x, y) \\
=\int\left(\mu_{x,\left(i, k^{(i)}\right)}(\cdot)-\mu_{y,\left(j, k^{(j)}\right)}(\cdot)\right)^{+}(d z) \\
\quad \times[h(x+z, y)-h(x, y)] \\
\quad+\int\left(\mu_{y,\left(j, k^{(j)}\right)}(\cdot)-\mu_{x,\left(i, k^{(i)}\right)}(\cdot)\right)^{+}(d z) \\
\quad \times[h(x, y+z)-h(x, y)] \\
\quad+\int\left(\mu_{x,\left(i, k^{(i)}\right)}(\cdot) \wedge \mu_{y,\left(j, k^{(j)}\right)}(\cdot)\right)(d z) \\
\quad \times[h(x+z, y+z)-h(x, y)],
\end{aligned}
$$

then the operator $\widetilde{\Omega}\left(\left(i, k^{(i)}\right),\left(j, k^{(j)}\right)\right)$ is a coupling of $\Omega\left(\left(i, k^{(i)}\right)\right)$ and $\Omega\left(\left(j, k^{(j)}\right)\right)$.

Next, we denote the transition probability family of the process $(X(t), r(t))$ by $\left\{P\left(t,\left(x,\left(i, k^{(i)}\right)\right), A\right): \quad t \geq\right.$ $\left.0,\left(x,\left(i, k^{(i)}\right)\right) \in R^{d} \times G, A \in \mathscr{B}\left(R^{d} \times G\right)\right\}$. For a subsequent use, we now introduce the Wasserstein metric between two probability measures as follows. For two probability measures $P_{1}$ and $P_{2}$, define

$$
\begin{aligned}
W\left(P_{1}, P_{2}\right)=\inf _{\Pi} \int_{R^{d} \times R^{d}} \lambda\left(\left(x,\left(i, k^{(i)}\right)\right),\left(y,\left(j, k^{(j)}\right)\right)\right) \\
\quad \times \Pi\left(d x, d\left(i, k^{(i)}\right), d y, d\left(j, k^{(j)}\right)\right),
\end{aligned}
$$

where $\Pi$ varies over all coupling probability measures with martingales $P_{1}$ and $P_{2}$. In order to prove the Feller continuity of $(X(t), \widehat{r}(t))$, we need to make the following assumption.

Assumption 11. Assume that there exists $H^{\prime}>0$ such that

$$
\int_{R^{d} \backslash\{0\}}|z|\left\|\mu_{x,\left(i, k^{(i)}\right)}(\cdot)-\mu_{y,\left(i, k^{(i)}\right)}(\cdot)\right\|(d z) \leq H^{\prime}|x-y|
$$

for all $x, y \in R^{d}$ and $\left(i, k^{(i)}\right) \in G$, where $\|\cdot\|$ denotes the total variation norm. 
Theorem 12. Let $(X(t), \widehat{r}(t))$ be the solution to the system given by (5) with initial value $(X(0), \widehat{r}(0))=(x, i)$. Assume that Assumptions 6 and 11 hold. Then for any bounded continuous function $\widehat{f}(\cdot, \cdot) \in R^{d} \times E \rightarrow R$, the function $u(x, \cdot)=$ $E^{(x, i)} \widehat{f}(X(t), \widehat{r}(t))$ is continuous with respect to $x$.

Proof. Recall that the $r(t)$ is a Markov process associated with the phase semi-Markov process $\widehat{r}(t)$. Hence $u(x, \cdot)=$ $E^{(x, i)} \widehat{f}(X(t), \widehat{r}(t))=E^{\left(x,\left(i, k^{(i)}\right)\right)} f(X(t), r(t))$, where $\widehat{f}(x, i) \triangleq$ $\widehat{f}\left(x,\left(i, k^{(i)}\right)\right)$. To prove the Feller property for $(X(t), \widehat{r}(t))$, it is equivalent to show Feller property for $(X(t), r(t))$ with respect to $x$. Since $G$ has the discrete metric, we only need to prove that, for any $t \geq 0, x, y \in R^{d}$, and $\left(i, k^{(i)}\right) \in G$, $P\left(t,\left(x,\left(i, k^{(i)}\right)\right), \cdot\right)$ converges weakly to $P\left(t,\left(y,\left(i, k^{(i)}\right)\right), \cdot\right)$ as $x \rightarrow y$. To this end, by means of Theorem 5.6 in Chen [12], it suffices to prove that

$$
\begin{array}{r}
W\left(P\left(t,\left(x,\left(i, k^{(i)}\right)\right), \cdot\right), P\left(t,\left(y,\left(i, k^{(i)}\right)\right), \cdot\right)\right) \\
\text { as } x \rightarrow 0
\end{array}
$$

We now use the coupling constructed in (25) to prove (33). Let $\left(X(t), r(t), Y(t), r^{\prime}(t)\right)$ denote the process corresponding to the coupling generator $\widetilde{\mathscr{A}}$, let $P^{\left(x,\left(i, k^{(i)}\right), y,\left(j, k^{(j)}\right)\right)}$ denote the distribution of $\left(X(t), r(t), Y(t), r^{\prime}(t)\right)$ starting from $\left(x,\left(i, k^{(i)}\right), y,\left(j, k^{(j)}\right)\right)$, and let $E^{\left(x,\left(i, k^{(i)}\right), y,\left(j, k^{(j)}\right)\right)}$ denote the corresponding expectation. Set

$$
\begin{aligned}
& S_{h}=\inf \left\{t \geq 0:\left|X_{t}-Y_{t}\right|>h\right\}, \quad h>1, \\
& T_{R}=\inf \left\{t \geq 0:\left|X_{t}\right|^{2}+\left|Y_{t}\right|^{2}>R\right\}, \quad V=T_{R} \wedge S_{h} .
\end{aligned}
$$

Using Dynkin's formula and noting that $r(t)=r^{\prime}(t)$ for all $t \geq 0$ whenever $r(0)=r^{\prime}(0)=\left(i, k^{(i)}\right)$, we have

$$
\begin{aligned}
& E^{\left(x,\left(i, k^{(i)}\right), y,\left(i, k^{(i)}\right)\right)} \lambda\left(\left(X_{t \wedge V}, r_{t \wedge V}\right),\left(Y_{t \wedge V}, r_{t \wedge V}^{\prime}\right)\right) \\
& =|x-y|+E^{\left(x,\left(i, k^{(i)}\right), y,\left(i, k^{(i)}\right)\right)} \int_{0}^{t \wedge V} \widetilde{\mathscr{A}} \lambda\left(\left(X_{u}, r_{u}\right),\left(Y_{u}, r_{u}^{\prime}\right)\right) d u \\
& =|x-y|+E^{\left(x,\left(i, k^{(i)}\right), y,\left(i, k^{(i)}\right)\right)} \int_{0}^{t \wedge V}\left(\widetilde{L}\left(r_{u}, r_{u}^{\prime}\right)\left|X_{u}-Y_{u}\right|\right. \\
& \left.+\widetilde{\Omega}\left(r_{u}, r_{u}^{\prime}\right)\left|X_{u}-Y_{u}\right|\right) d u
\end{aligned}
$$

for $x, y \in R^{d}$ and $\left(i, k^{(i)}\right) \in G$.

Next, we give some estimation on the integrand of the rightmost term in (35). It follows from (18) in Chen and Li [11] that

$$
\begin{aligned}
& \widetilde{L}\left(\left(i, k^{(i)}\right),\left(i, k^{(i)}\right)\right)|x-y| \\
& \quad \leq \frac{\left[\operatorname{tr} A\left(x, y,\left(i, k^{(i)}\right)\right)+2 \widehat{B}\left(x, y,\left(i, k^{(i)}\right)\right)\right]}{2|x-y|},
\end{aligned}
$$

where

$$
\begin{aligned}
& A\left(x, y,\left(i, k^{(i)}\right)\right)= a\left(x,\left(i, k^{(i)}\right)\right)+a\left(y,\left(i, k^{(i)}\right)\right) \\
&-2 \sigma\left(x,\left(i, k^{(i)}\right)\right) \sigma^{*}\left(y,\left(i, k^{(i)}\right)\right), \\
& \widehat{B}\left(x, y,\left(i, k^{(i)}\right)\right)=\left\langle x-y, b\left(x,\left(i, k^{(i)}\right)\right)-b\left(y,\left(i, k^{(i)}\right)\right)\right\rangle .
\end{aligned}
$$

Moreover,

$$
\begin{aligned}
\operatorname{tr} A\left(x, y,\left(i, k^{(i)}\right)\right) & =\left|\sigma\left(x,\left(i, k^{(i)}\right)\right)-\sigma\left(y,\left(i, k^{(i)}\right)\right)\right|^{2} \\
2 \widehat{B}\left(x, y,\left(i, k^{(i)}\right)\right) \leq & |x-y|^{2} \\
& +\left|b\left(x,\left(i, k^{(i)}\right)\right)-b\left(y,\left(i, k^{(i)}\right)\right)\right|^{2} .
\end{aligned}
$$

Therefore, from (36) and Proposition 9, one has

$$
\widetilde{L}\left(\left(i, k^{(i)}\right),\left(i, k^{(i)}\right)\right)|x-y| \leq \frac{H+1}{2}|x-y|,
$$

for $x, y \in R^{d}$ and $\left(i, k^{(i)}\right) \in G$. With some straightforward calculations, we have

$$
\begin{aligned}
& \widetilde{\Omega}\left(\left(i, k^{(i)}\right),\left(i, k^{(i)}\right)\right)|x-y| \\
& =\int\left(\mu_{x,\left(i, k^{(i)}\right)}(\cdot)-\mu_{y,\left(i, k^{(i)}\right)}(\cdot)\right)^{+}(d z)(|x+z-y|-|x-y|) \\
& \quad+\int\left(\mu_{y,\left(i, k^{(i)}\right)}(\cdot)-\mu_{x,\left(i, k^{(i)}\right)}(\cdot)\right)^{+}(d z)(|x-y-z|-|x-y|) \\
& \leq \int|z|\left\|\mu_{x,\left(i, k^{(i)}\right)}(\cdot)-\mu_{y,\left(i, k^{(i)}\right)}(\cdot)\right\|(d z) \leq H^{\prime}|x-y|
\end{aligned}
$$

for $x, y \in R^{d}$ and $\left(i, k^{(i)}\right) \in G$. Now substituting (39) and (40) into (35), we arrive at

$$
\begin{aligned}
& E^{\left(x,\left(i, k^{(i)}\right), y,\left(i, k^{(i)}\right)\right)} \lambda\left(\left(X_{t \wedge V}, r_{t \wedge V}\right),\left(Y_{t \wedge V}, r_{t \wedge V}^{\prime}\right)\right) \\
& \leq|x-y|+\frac{2 H^{\prime}+H+1}{2} \\
& \quad \times \int_{0}^{t} E^{\left(x,\left(i, k^{(i)}\right), y,\left(i, k^{(i)}\right)\right)}\left|X_{u \wedge V}-Y_{u \wedge V}\right| d u \\
& =|x-y|+\frac{2 H^{\prime}+H+1}{2} \\
& \quad \times \int_{0}^{t} \lambda\left(\left(X_{u \wedge V}, r_{u \wedge V}\right),\left(Y_{u \wedge V}, r_{u \wedge V}^{\prime}\right)\right) d u .
\end{aligned}
$$

Thus, by Gronwall's inequality, we obtain

$$
\begin{gathered}
E^{\left(x,\left(i, k^{(i)}\right), y,\left(i, k^{(i)}\right)\right)} \lambda\left(\left(X_{t \wedge V}, r_{t \wedge V}\right),\left(Y_{t \wedge V}, r_{t \wedge V}^{\prime}\right)\right) \\
\leq|x-y| \exp \left(\frac{\left(2 H^{\prime}+H+1\right) t}{2}\right)
\end{gathered}
$$


Finally, letting $R \uparrow \infty$ and $h \uparrow \infty$, we conclude that

$$
\begin{aligned}
& E^{\left(x,\left(i, k^{(i)}\right), y,\left(i, k^{(i)}\right)\right)} \lambda\left((X(t), r(t)),\left(Y(t), r^{\prime}(t)\right)\right) \\
& \quad \leq|x-y| \exp \left(\frac{\left(2 H^{\prime}+H+1\right) t}{2}\right)
\end{aligned}
$$

which implies (33). The proof is complete.

\section{Strong Feller Continuity}

In the following we will prove the strong Feller continuity of the process $(X(t), \widehat{r}(t))$ by using the relation between the transition probability of jump-diffusion and the corresponding diffusions in Skorohod [13]. First we introduce some auxiliary processes as follows. For each $\left(i, k^{(i)}\right) \in G$, let $X_{\left(i, k^{(i)}\right)}^{0}(t)$ be the unique strong solution to the following stochastic differential equation in $R^{d}$ :

$$
\begin{aligned}
d X_{\left(i, k^{(i)}\right)}^{0}(t)= & \sigma\left(X_{\left(i, k^{(i)}\right)}^{0}(t),\left(i, k^{(i)}\right)\right) d B(t) \\
& +b\left(X_{\left(i, k^{(i)}\right)}^{0}(t),\left(i, k^{(i)}\right)\right) d t .
\end{aligned}
$$

For each $\left(i, k^{(i)}\right) \in G$, we denote the transition probability families of the jump-diffusion $X_{\left(i, k^{(i)}\right)}(t)$ satisfying $(20)$ when $r(t)=\left(i, k^{(i)}\right)$ and the diffusion $X_{\left(i, k^{(i)}\right)}^{0}(t)$ by $\left\{P^{\left(i, k^{(i)}\right)}(t, x, A)\right.$ : $\left.t \geq 0, x \in R^{d}, A \in \mathscr{B}\left(R^{d}\right)\right\}$ and $\left\{P^{\left(i, k^{(i)}\right), 0}(t, x, A): t \geq 0, x \in\right.$ $\left.R^{d}, A \in \mathscr{B}\left(R^{d}\right)\right\}$, respectively.

Lemma 13. For any given $\left(i, k^{(i)}\right) \in G$, if the transition probability $P^{\left(i, k^{(i)}\right), 0}(t, x, A)$ has a density $P^{\left(i, k^{(i)}\right), 0}(t, x, y)$ with respect to the Lebesgue measure, then the transition probability $P^{\left(i, k^{(i)}\right)}(t, x, A)$ also has a density $P^{\left(i, k^{(i)}\right)}(t, x, y)$ with respect to Lebesgue measure. Moreover, if the transition probability density $P^{\left(i, k^{(i)}\right), 0}(t, x, y)$ is positive, then the transition probability density $P^{\left(i, k^{(i)}\right)}(t, x, y)$ also is positive.

Proof. Please see Chapter 1 in Skorohod [13].

Assumption 14. For each $\left(i, k^{(i)}\right) \in G$, assume that the diffusion $X_{\left(i, k^{(i)}\right)}^{0}(t)$ determined by $(44)$ has a positive probability density $P^{\left(\left(i, k^{(i)}\right)\right), 0}(t, x, y)$ with respect to the Lebesgue measure.

To prove the strong Feller continuity, we need to prove that, for every $t>0$ and every $\left(x,\left(i, k^{(i)}\right)\right) \in R^{d} \times G$, the transition probability $P\left(t,\left(x,\left(i, k^{(i)}\right)\right), \cdot\right)$ corresponding to $(X(t), r(t))$ is absolutely continuous with respect to a reference measure $\tilde{\mu}(\cdot)$. Here and hereafter, the reference measure $\tilde{\mu}(\cdot)$ is the product measure on $R^{d} \times G$ of the Lebesgue measure on $R^{d}$ and the counting measure on $G$. In addition, the Lebesgue measure on $R^{d}$ will be denoted by $m(\cdot)$. Hereafter, for a given set $D \in \mathscr{B}\left(R^{d} \times G\right)$, let, for each $\left(i, k^{(i)}\right) \in G$,

$$
D_{\left(i, k^{(i)}\right)}=\left\{x \in R^{d}:\left(x,\left(i, k^{(i)}\right)\right) \in D\right\}
$$

be the section of $D$ at $\left(i, k^{(i)}\right)$. Then, the product measure theorem gives us the following lemma which will be used later.

Lemma 15. For any set $D \in \mathscr{B}\left(R^{d} \times G\right)$ with $\tilde{\mu}(D)=0$, we have that $m\left(D_{\left(i, k^{(i)}\right)}\right)=0$ for all $\left(i, k^{(i)}\right) \in G$.

Recall that $N(t)=\max \left\{n: \tau_{n} \leq t\right\}$. Therefore, for every $\left(x,\left(i, k^{(i)}\right)\right) \in R^{d} \times G$, every $t>0$, and every set $D \in \mathscr{B}\left(R^{d} \times G\right)$, we have that

$$
\begin{aligned}
& P^{\left(x,\left(i, k^{(i)}\right)\right)}((X(t), r(t)) \in D) \\
& \quad=\sum_{n=0}^{\infty} P^{\left(x,\left(i, k^{(i)}\right)\right)}((X(t), r(t)) \in D, N(t)=n) .
\end{aligned}
$$

Lemma 16. Suppose that Assumption 14 holds. One has the following.

(i) For every $\left(x,\left(i, k^{(i)}\right)\right) \in R^{d} \times G$, every $t>0$, and every integer $n \geq 0$, we have that

$$
P^{\left(x,\left(i, k^{(i)}\right)\right)}((X(t), r(t)) \in D, N(t)=n)=0
$$

for any set $D \in \mathscr{B}\left(R^{d} \times G\right)$ satisfying $\widetilde{\mu}(D)=0$.

(ii) For every $\left(x,\left(i, k^{(i)}\right)\right) \in R^{d} \times G$ and every $t>0$, we have that

$$
P^{\left(x,\left(i, k^{(i)}\right)\right)}((X(t), r(t)) \in D)=0
$$

$$
\text { for any set } D \in \mathscr{B}\left(R^{d} \times G\right) \text { satisfying } \widetilde{\mu}(D)=0 \text {. }
$$

The following theorem is our main result in this section.

Theorem 17. Suppose that Assumptions 11 and 14 hold. For every $\left(x,\left(i, k^{(i)}\right)\right) \in R^{d} \times G$ and every $t>0$, the transition probability $P\left(t,\left(x,\left(i, k^{(i)}\right)\right), \cdot\right)$ is absolutely continuous with respect to $\mu(\cdot)$. Then, for any bounded measurable function $\widehat{f}(\cdot, \cdot) \in R^{d} \times E \rightarrow R$, the function $u(x, \cdot)=E^{(x, i)} \widehat{f}(X(t), \widehat{r}(t))$ is continuous with respect to $x$.

Proof. The former assertion readily follows from Lemma 16. In the following we prove the latter assertion. For a set $A \in \mathscr{B}\left(R^{d} \times G\right)$, let $\bar{A}, A^{\circ}$, and $\partial A$ denote the closure, the interior, and the boundary of $A$, respectively. Following from the definition of the reference measure, it is easy to get that $\tilde{\mu}(\partial A)=0$ for all $A \in \mathscr{B}\left(R^{d} \times G\right)$. Therefore, by virtue of the absolute continuity and the Feller continuity proved in 
Theorem 12 and Lemma 16, we can conclude that, for every $\left(x,\left(i, k^{(i)}\right)\right) \in R^{d} \times G$ and every $t>0$,

$$
\begin{aligned}
& \lim _{y \rightarrow x} \inf P\left(t,\left(y,\left(i, k^{(i)}\right)\right), A\right) \\
& \geq \lim _{y \rightarrow x} \inf P\left(t,\left(y,\left(i, k^{(i)}\right)\right), A^{\circ}\right) \\
& \geq P\left(t,\left(x,\left(i, k^{(i)}\right)\right), A^{\circ}\right)=P\left(t,\left(x,\left(i, k^{(i)}\right)\right), A\right)
\end{aligned}
$$

which implies the desired strong Feller continuity. The proof is therefore complete.

\section{Conclusion}

In this paper, we discuss the stochastic stability of a more general jump-diffusion process with phase semi-Markovian switching. We first transform it into a Markov process by the way of supplementary variable technique. Then using coupling method, we verify that, under linear growth and some proper conditions, the jump-diffusion with phase semiMarkovian switching keeps Feller continuous and strong Feller continuous. However, it is worthy to study some other properties (recurrence property, existence and uniqueness for the stationary distribution) for the process defined by (5).

\section{Conflict of Interests}

The author declares that there is no conflict of interests regarding the publication of this paper.

\section{Acknowledgments}

The author is grateful to the anonymous referees for their valuable comments and suggestions which led to improvements in this paper. Research of the author was partially supported by National Natural Science Foundation of China (nos. 11171062, 11101077 and 11201062), the Fundamental Research Funds for the Central Universities.

\section{References}

[1] X. R. Mao, "Stability of stochastic differential equations with Markovian switching," Stochastic Processes and Their Applications, vol. 79, no. 1, pp. 45-67, 1999.

[2] L. Shaikhet, "Stability of stochastic hereditary systems with Markov switching," Theory of Stochastic Processes, vol. 2, no. 18, pp. 180-184, 1996.

[3] X. R. Mao, A. Matasov, and A. B. Piunovskiy, "Stochastic differential delay equations with Markovian switching," Bernoulli, vol. 6, no. 1, pp. 73-90, 2000.

[4] I.-S. Wee, "Stability for multidimensional jump-diffusion processes," Stochastic Processes and Their Applications, vol. 80, no. 2, pp. 193-209, 1999.

[5] C. G. Yuan and X. R. Mao, "Asymptotic stability in distribution of stochastic differential equations with Markovian switching," Stochastic Processes and Their Applications, vol. 103, no. 2, pp. 277-291, 2003.
[6] Z. Z. Zhang and D. Y. Chen, "A new criterion on existence and uniqueness of stationary distribution for diffusion processes," Advances in Difference Equations, vol. 13, pp. 1-6, 2013.

[7] F. B. Xi, "Stability of a random diffusion with nonlinear drift," Statistics \& Probability Letters, vol. 68, no. 3, pp. 273-286, 2004.

[8] F. B. Xi, "On the stability of jump-diffusions with Markovian switching," Journal of Mathematical Analysis and Applications, vol. 341, no. 1, pp. 588-600, 2008.

[9] M. F. Neuts, "Computational uses of the method of phases in the theory in the theory of queues," Computers \& Mathematics with Applications, vol. 1, pp. 151-166, 1975.

[10] N. Ikeda and S. Watanabe, Stochastic Differential Equations and Diffusion Processes, North-Holland, Amsterdam, The Netherlands, 1989.

[11] M. F. Chen and S. F. Li, "Coupling methods for multidimensional diffusion processes," The Annals of Probability, vol. 17, no. 1, pp. 151-177, 1989.

[12] M.-F. Chen, From Markov Chains to Non-Equilibrium Particle Systems, World Scientific, Singapore, 2004.

[13] A. V. Skorokhod, Asymptotic Methods in the Theory of Stochastic Differential Equations, American Mathematical Society, Providence, RI, USA, 1989. 


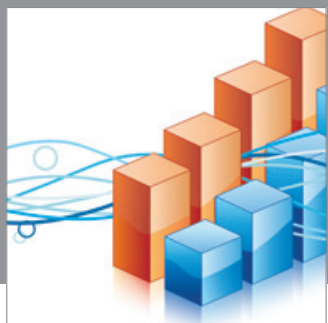

Advances in

Operations Research

mansans

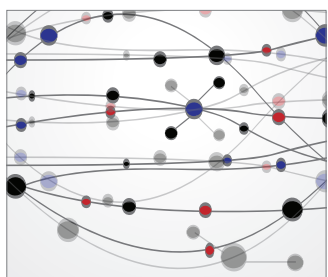

The Scientific World Journal
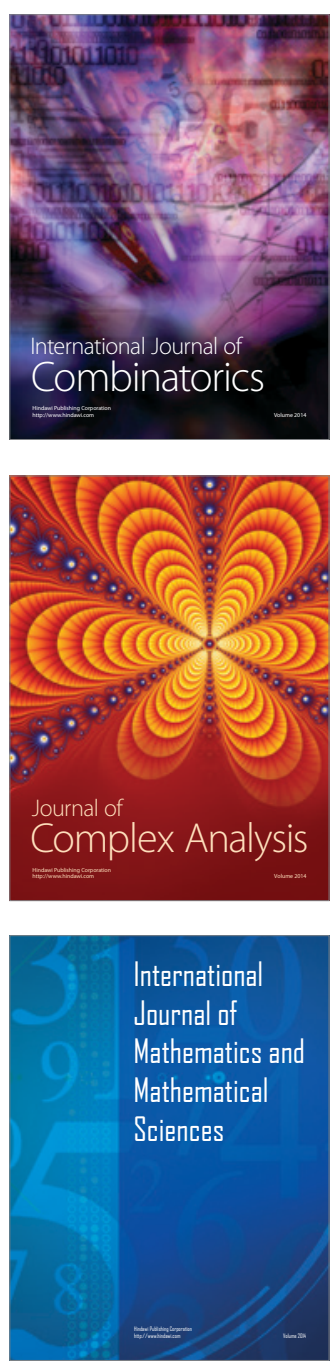
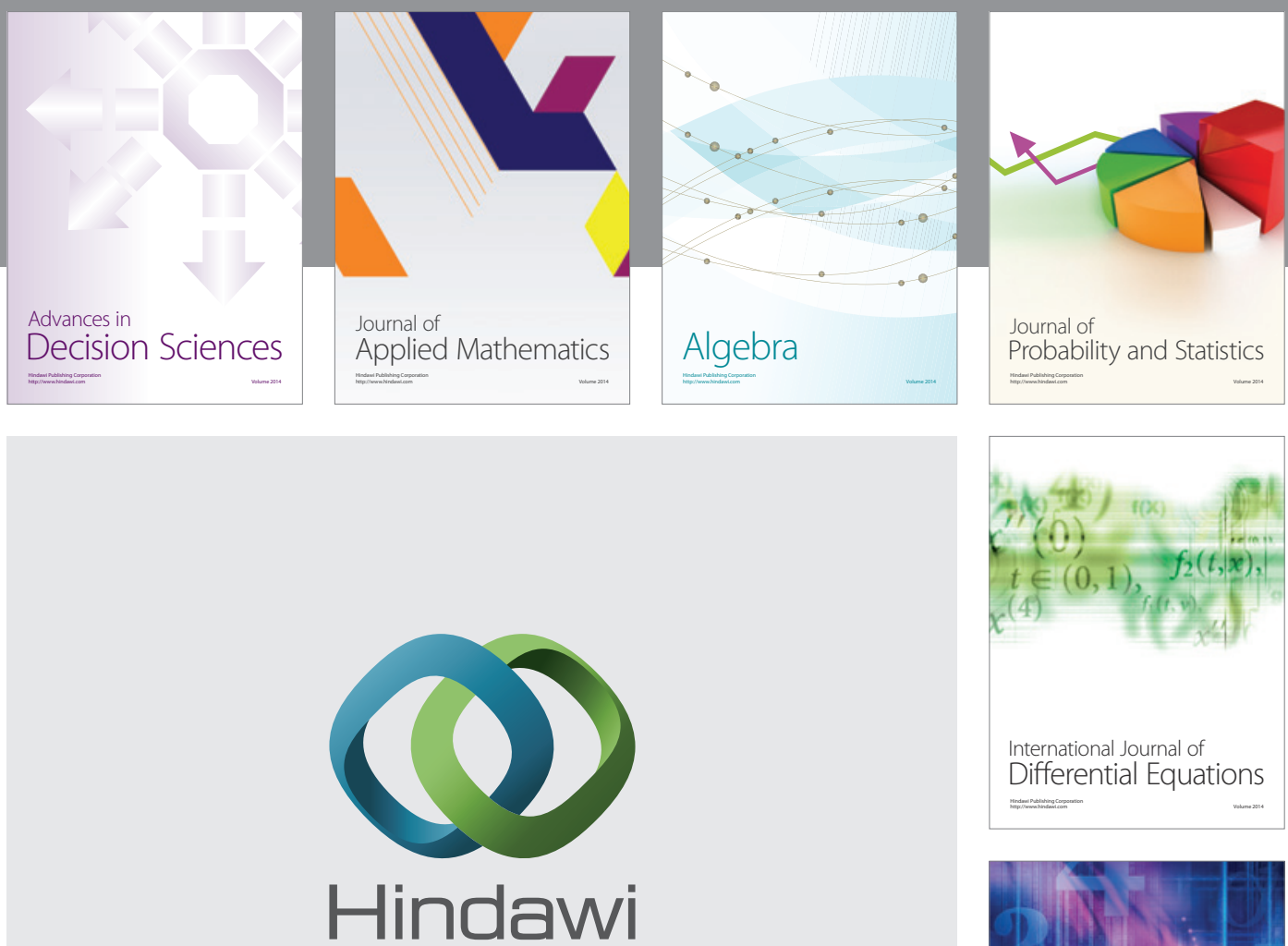

Submit your manuscripts at http://www.hindawi.com
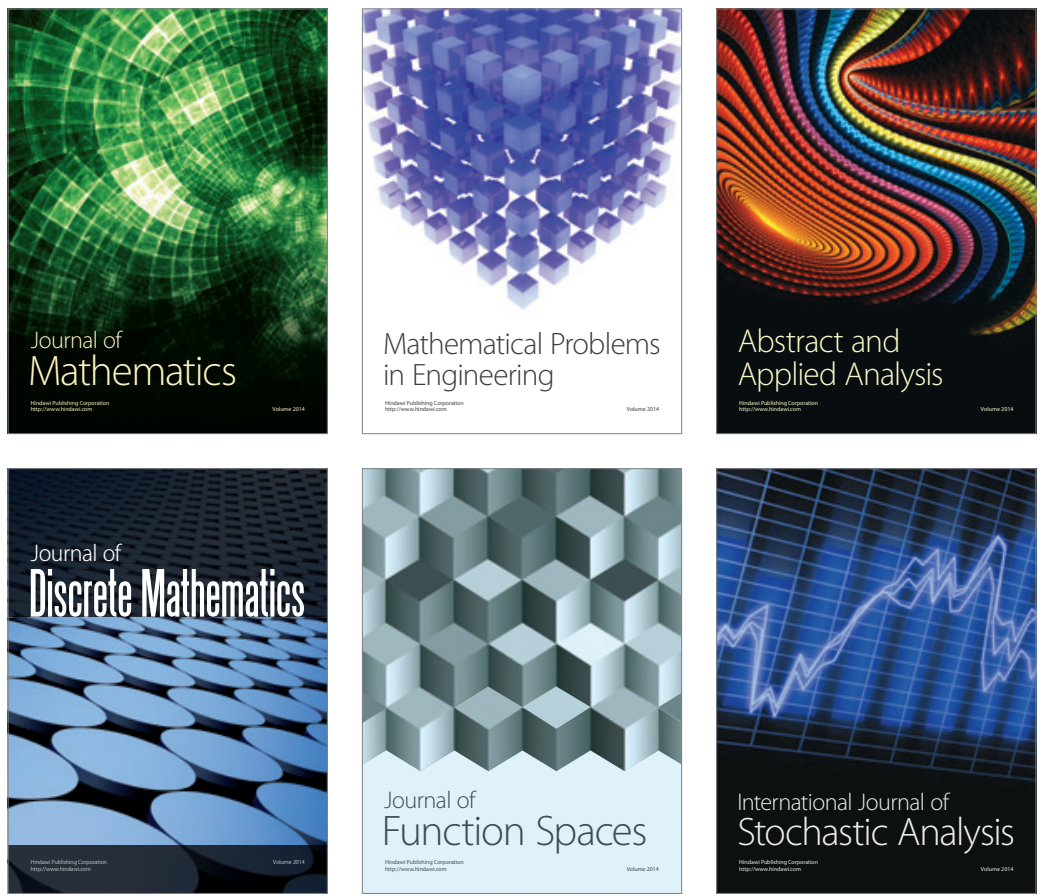

Journal of

Function Spaces

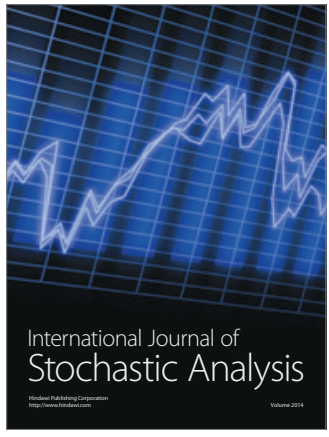

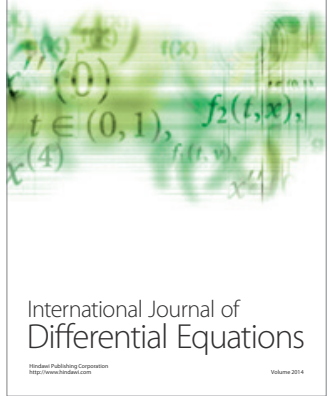
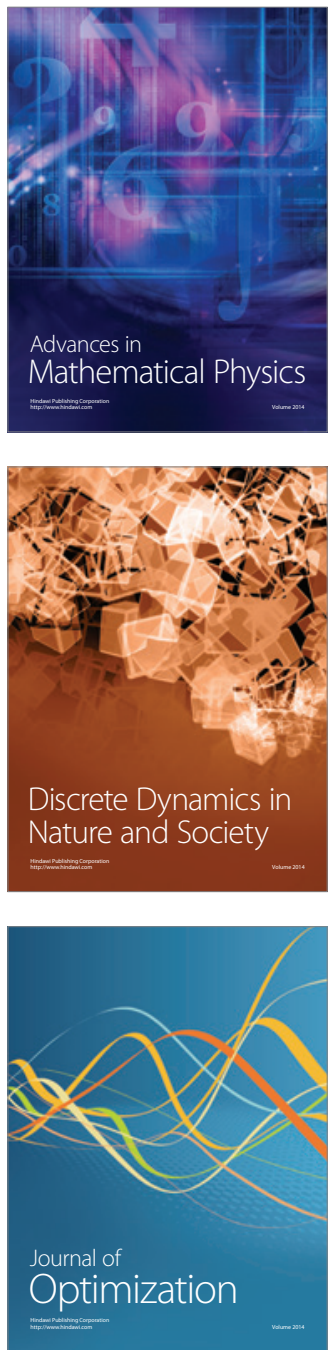